\title{
Bracing of Thoracic and Lumbar Spine Fractures
}

\author{
R. F. Jones, F.R.A.C.S., D.P.R.M., F.R.A.C.P., F.A.C.R.M., ${ }^{1}$ Elizabeth \\ Snowdon, M.C.S.P., ${ }^{2}$ J. Coan, M.A.O.P.A., ${ }^{3}$ Linda King, Dip.Physio- \\ therapy, Stella Engel, M.B.B.S., D.P.R.M., F.A.C.R.M. ${ }^{4}$ \\ ${ }^{1}$ Director, ${ }^{2}$ Physiotherapist, ${ }^{3}$ Orthotist,${ }^{4}$ Associate Director, The Spinal Injury Unit \\ and Department of Rehabilitation, The Prince Henry Hospital, University of New \\ South Wales, Sydney, Australia
}

\section{Summary}

A prospective study of the non-operative management of 33 thoracic and lumbar fractures and dislocations was undertaken examining factors such as duration of bed rest and brace prescription. Outcome was assessed using Frankel's grading system and serial radiological examination.

Bed rest for 6 to 8 weeks followed by 16 weeks of immobilisation in a thoracolumbar orthosis (either a moulded plastazote lined polythene brace or a Taylor brace) resulted in less than $15^{\circ}$ kyphus in $85^{\circ}{ }^{\circ}$ of patients and less than $20^{\circ}{ }_{0}$ kyphus in $94^{\circ}{ }^{\circ}$. One patient with a T12/L1 dislocation who had an early decompressive laminectomy showed persistent instability and required internal fixation. One patient with an L1 fracture who initially wore a Hexalite brace developed late instability (at 12 months after injury) and required spinal osteotomy and fusion. Neurological improvement was observed in $50^{\circ}{ }_{0}$ of patients, and no permanent neurological deterioration occurred.

A non-operative regimen of treatment of fractured thoracic and lumbar vertebrae as described is an alternative to prolonged immobilisation in bed, or early operative fusion.

Key words: Spinal fractures-thoracic and lumbar; Conservative (non-operative) management; Orthosis; Brace; Outcome.

\section{Introduction}

Little consensus exists regarding the early management of fractures of thoracic and lumbar vertebrae with or without spinal cord injury (Ryan and Taylor, 1982). Treatment must take into account the requirements of the fracture as well as the state of the spinal cord. In complete cord lesions, one looks toward as early a mobilisation as possible in order to prevent or minimise the morbidity associated with prolonged recumbency. Early mobilisation without adequate internal or external fixation however may allow an unacceptable kyphus to develop at the site of the fracture, a deformity which is functionally and cos- 
metically poor. In patients with an incomplete neurological lesion, early activity without stability may compromise a spinal canal already narrowed by haematoma, oedema or bony fragment, and even cause further trauma to the cord either directly or indirectly by ischaemia. Where no neurological damage has occurred, treatment must minimise the disruption caused by the patient's absence from family and job as well as ensuring satisfactory healing in good position.

Prolonged periods of bed rest, sometimes in excess of 6 months used to be advised for the management of fractures of major elements of thoracic and lumbar vertebrae (reference Guttmann 1976). Despite fears that instability and deformities would occur, the time for immobilisation for these fractures has gradually been reduced, and mobilisation with external bracing has been employed far earlier than previously. In a few patients indications for early internal fixation exist including gross disruption and progressive neurological deficit. The risks and benefits of internal fixation for vertebral fractures should be weighed against those of a non-operative approach. Bedbrook (1985) clearly outlines the case for non-operative care whilst conceding certain indications for operative intervention. Our paper describes a prospective study of non-operative techniques used in the management of thoracic and lumbar fractures and dislocations.

\section{Patients and methods}

Thirty three consecutive patients with thoracic or lumbar fractures admitted to the Spinal Injury Unit at The Prince Henry Hospital were studied. Twenty seven suffered neurological loss and six had no neurological loss. Both groups were treated in a similar fashion with respect to their fracture, nursing and brace prescription. A non-operative approach consisting of reduction of the fracture by posture and second hourly skin pressure relief by lifting, log rolling or turning with an Egerton Stoke-Mandeville bed was maintained for a period of 6 to 8 weeks. When acute back pain had abated or was controlled with analgesics the patients were taught to perform isometric back exercises several times daily on their back. This regimen was similar to that advocated by Bedbrook (Bedbrook 1975). Radiological examination with fluoroscopy was performed to assess healing and stability at the end of this period. If instability was not detected the patient was mobilised with a brace for a further 16 weeks. Radiographic views of the fracture in flexion and extension were then performed without the brace in order to assess stability. If stability was demonstrated the brace was discarded. Six months after brace removal the radiological examination in flexion and extension was repeated.

The extent of vertebral fracture was assessed by antero-posterior and lateral radiographs. They were classified into three categories.

Category one included those fractures where there was less than one third compression of the vertebra and less than 1 centimetre antero-posterior displacement. Category two included fractures where there was greater than one third compression with 1 centimetre or more of displacement, but less severe than category three which included those fractures where there was severe disruption 
PARA PLEG I A

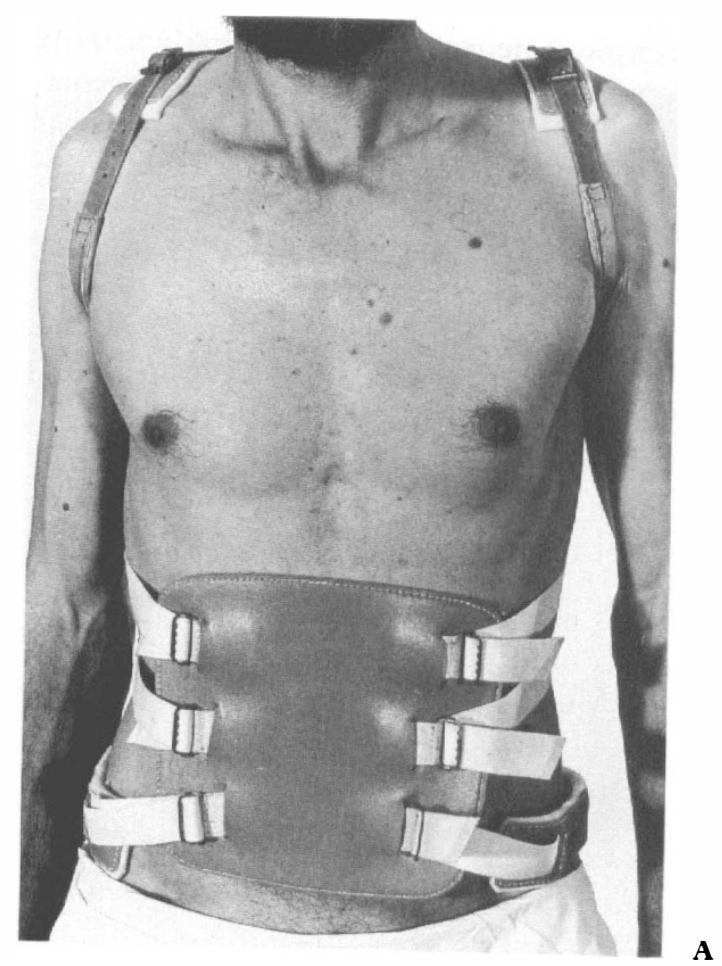

Figure 1A,B - Taylor Brace without groin strap.

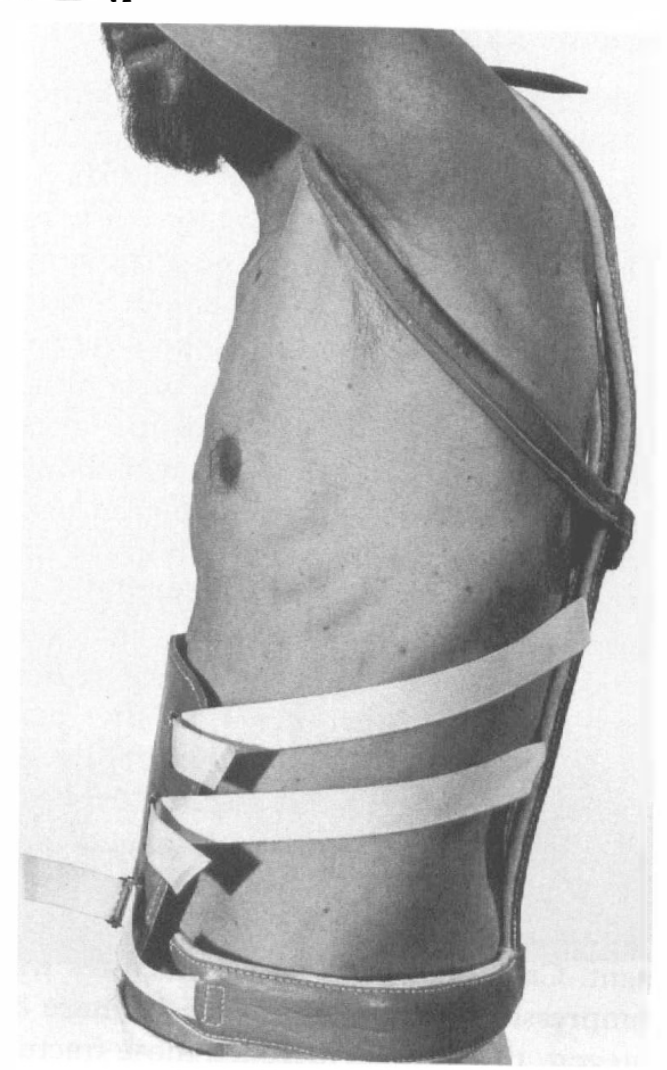



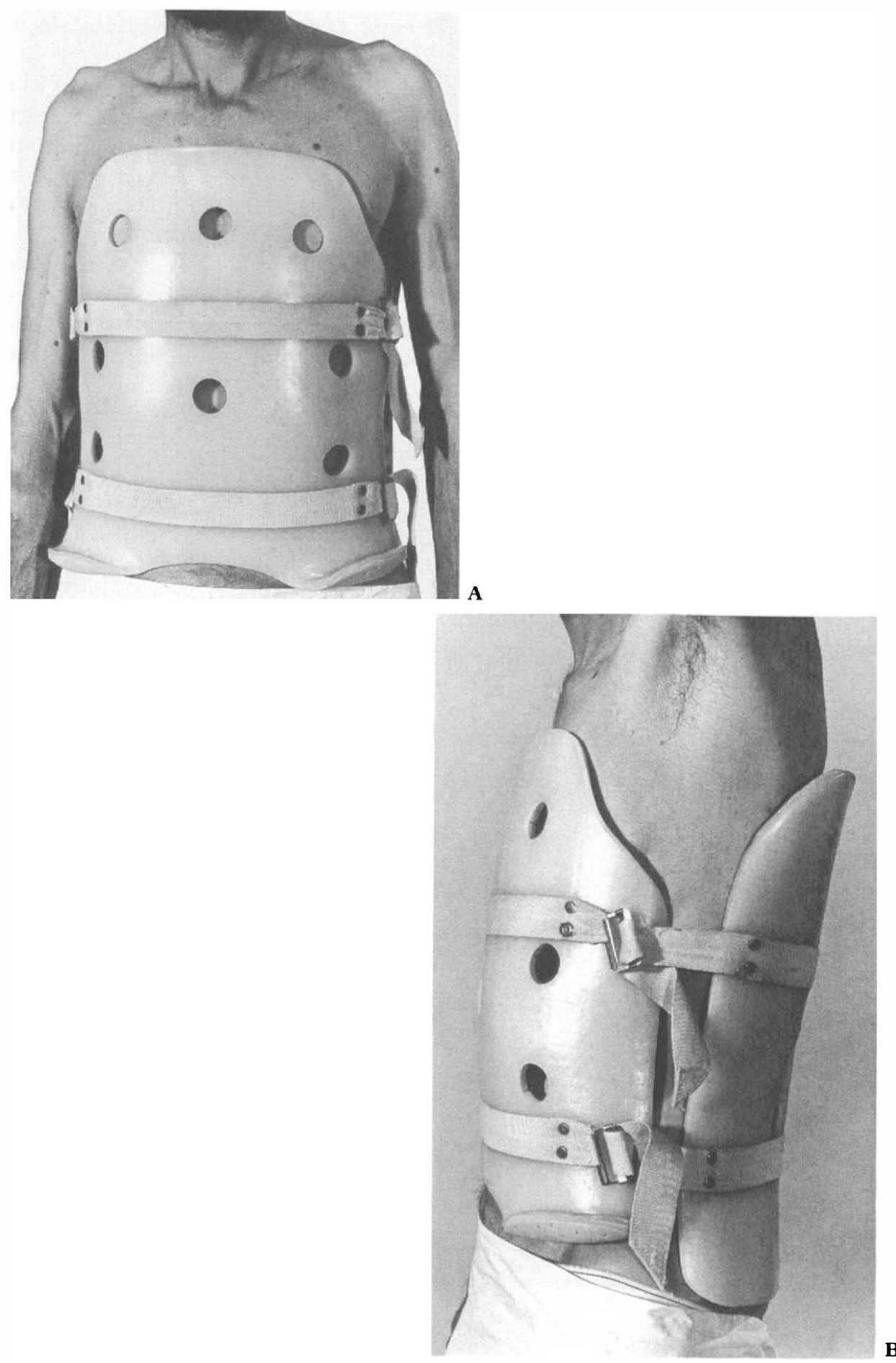

Figure 2A,B - Plastazote lined moulded polythene brace. 
of the vertebral body, severe posterior element damage and gross displacement of the fragments.

If neurological deterioration occurred in an incomplete cord lesion, or if in a cauda equina lesion recovery was insufficient for ambulation, decompressive laminectomy was considered. Myelography and computerised tomography were used as diagnostic aids. If there was a block to the flow of dye then operation was performed. If lacerated nerve roots were discovered then on the basis that a peripheral nerve injury existed, suturing was performed.

The braces (Fig. 1, Fig. 2) were made by the orthotists in the Department of Rehabilitation. Litecast, Hexalite, Taylor and moulded Plastazote-lined polythene braces (PLP braces) were used initially. Problems of fitting became evident with Litecast and Hexalite, so subsequently Taylor and PLP braces were used exclusively.

Because of the inability to mould a PLP brace higher than the T4/5 region, their use was restricted to fractures below that level. Taylor braces were used without groin straps so as to minimise the risk of pressure necrosis in patients with neurological loss; occasionally this resulted in difficulty in maintaining the caudal location of the brace. Patients changed into a waterproof brace for showering.

PLP braces took approximately 3 hours to make and required 45 minutes of fitting time and the services of two people during the fitting. The brace was bivalved for easy removal. The materials were not water absorbent.

Taylor braces required 15 minutes for measurement and 20 minutes for fitting with the services of two people.

Hexalite and Litecast braces were discontinued because of difficulty in maintaining the position of the brace and the inability to remove the brace easily once applied.

Assessment of neurological progress was made using Frankel's grading system (Frankel, Hancock and Hyslop, 1969). Fracture alignment was classified as excellent, good, fair or poor, depending on the radiological examination 1 year post fracture. Healing with up to $10^{\circ}$ of kyphus was termed excellent, between $10^{\circ}$ and $15^{\circ}$ was termed good, between $15^{\circ}$ and $20^{\circ}$ was termed fair and a poor result was kyphus beyond $20^{\circ}$.

\section{Results}

Four patients had fractures at the T3/4 level, thirteen between $\mathrm{T} 5$ and $\mathrm{T} 12$, nine at the T12/L1 level and seven at the L2/3 level (Table I).

Table I Distribution of Patients Äccording to Level of Fracture and Brace Prescribed

\begin{tabular}{lcccc}
\hline Level & Polythene & Taylor & Hexalite & Total \\
\hline T3/4 & 0 & 4 & 0 & 4 \\
T5/T12 & 8 & 5 & 0 & 13 \\
T12/L1 & 2 & 6 & 1 & 9 \\
L2/L3 & 4 & 0 & 3 & 7 \\
& & One Litecast & & \\
& changed & & \\
& to Taylor \\
\hline
\end{tabular}


A total of six patients with $\mathrm{T} 12 / \mathrm{L} 1$ fracture dislocation and one patient with an L2 fracture underwent decompressive laminectomy and then bed rest for 6 to 8 weeks. One patient with $\mathrm{T} 12 / \mathrm{L} 1$ fracture dislocation who had had early decompressive laminectomy showed persistent instability after 14 weeks and required internal fixation. One of the two patients who had a poor result required spinal osteotomy after 12 months.

Fourteen patients were fitted with a PLP brace, 15 were fitted with a Taylor brace, three with Hexalite and one with Litecast brace that was later changed to a Taylor brace.

The 14 patients using PLP braces showed eleven excellent results, three good and one fair result. Fifteen patients wearing Taylor braces recorded seven excellent (one later lost to follow up, showed an excellent result at 8 weeks), five good and one fair result. Those in Hexalite braces showed one good result and two poor results (Table II).

Table II Alignment results

\begin{tabular}{lcccc}
\hline $\begin{array}{l}\text { Degree of } \\
\text { Kyphus }\end{array}$ & $\begin{array}{c}\text { Excellent } \\
\text { less than 10 }\end{array}$ & $\begin{array}{c}\text { Good } \\
\text { less than 15 }\end{array}$ & $\begin{array}{c}\text { Fair } \\
\text { less than 20 }\end{array}$ & $\begin{array}{c}\text { Poor } \\
\text { greater than 20 }\end{array}$ \\
\hline $\begin{array}{l}\text { Polythene } \\
\text { Taylor }\end{array}$ & 11 & 2 & 1 & 0 \\
Hexalite & 8 & 6 & 2 & 0 \\
Litecast & 0 & 1 & 0 & 2 \\
\hline
\end{tabular}

Five patients fulfilled the category one fracture description, and all of these had an excellent result. Twenty two patients had category two fractures and of these, 14 had an excellent result, five had a good result, two a fair result and one a poor result. Six patients had a category three fracture and of these one had an excellent result, three had a good result, one had a fair result and one a poor result.

No patient deteriorated neurologically during the acute or follow up phase of the programme. Seventeen patients with incomplete lesions improved at least one grade of the Frankel table (Table III).

Patients in PLP braces complained more of restriction of movement than those in Taylor braces whilst PLP braces were found to be uncomfortable in the summer. Those in Taylor braces frequently complained of pressure on the top of the shoulders due either to inadequate shaping of the over-shoulder stems or to the arm straps being pulled too tightly. Overall those with the PLP braces had either excellent or good alignment.

Two patients $\left(6^{\circ}{ }_{0}\right)$ developed clinical deep venous thrombosis, one of these postoperatively following laminectomy. The other patient had suffered a severe head injury complicating the thoracic fracture and paraplegia.

No decubitus skin ulceration occurred. One patient had a urinary tract infection. Otherwise the presence of bacteriuria was unchanged from previous studies of urinary infection in the unit.

\section{Discussion}

This study shows that relatively simple braces can support the thoracic and lumbar spines in patients with or without neurological loss. Garzione and Clifton 
Table III Neurological progress

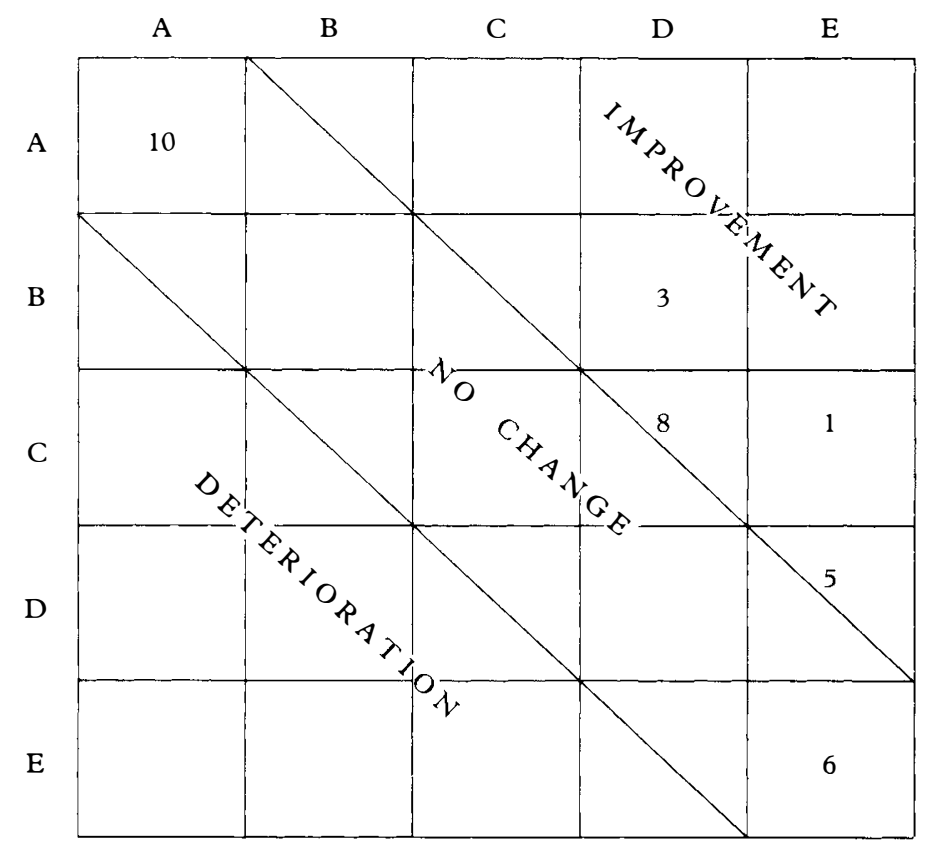

Assessment using Frankel's system:

A. Complete Loss: Complete loss of Motor and Sensory Function below the level of the lesion.

B. Sensory only: Some sensation present below the level of the lesion but complete motor paralysis.

C. Motor useless: Some motor power present below the lesion but insufficient for practical use.

D. Motor useful: Useful motor power below the level of the lesion.

E. Intact: No neurological deficit or symptoms.

(1976) who used Plastazote braces in elderly patients with fractured vertebrae and no neurological loss found that early mobilisation in an external orthosis can be a satisfactory non-operative regimen in elderly patients.

Nabel et al. (1963) working with cadaver specimens noted that the TaylorKnight brace was effective in limiting flexion-extension and lateral bending but not axial rotation. A body cast (material unstated) was shown to limit all motion in the cadaver but concern was expressed about the risks of skin damage in clinical application.

Contrary to the experience of Stauffer (1974) who found fractures in the lumbar spine difficult to immobilise by external orthoses, our four patients with L2/3 fractures managed with PLP braces had either excellent or good alignment.

A regimen of a brief period of rest and mobilisation without external bracing was reported by Harkonen et al. (1979), 89 cases sustained an anterior wedge fracture, 26 had two fractured vertebrae, seven had three fractured vertebrae, two had four fractured vertebrae, three had five fractured vertebrae, 26 patients had thoraco-lumbar fractures and three had sustained a lumbar fracture. Neuro- 
logical symptoms were present in nine patients $\left(9^{\circ}{ }_{0}\right)$, paraplegia developed in seven and an incomplete cord lesion or radicular symptoms developed in one patient with anterior wedge fracture and in one patient with fracture dislocation. The treatment consisted of rest and functional muscular exercise, three patients had a brace and two had a 'osteosynthesis'. The conservative bed rest group were mobilised from bed within 17 days on average, the range being from immediate to 179 days. Results were determined clinically, radiologically, and by subjective pain. None had neurological improvement and three deteriorated. The compression deformity was completely cured in $6^{\circ}{ }_{0}$ and increased in $6^{\circ}{ }_{0}$ who had a deformity of $\leqq 25^{\circ}$ o. All other radiological abnormalities increased during the time of observation. Härkönen et al. (1969) concluded that surgery as well as the use of an orthopaedic brace debilitate the back musculature causing a deleterious effect in the clinical result. The treatment used did not prevent deterioration in the radiological deformities although the authors decided that non-operative treatment of stable fractures seemed to be the method of choice.

Doubts about the efficacy of external bracing and the long period of convalescence prior to mobilisation have deterred some from adopting a conservative approach. We have concluded that bed rest for 6 to 8 weeks for fractures of the thoracic and lumbar vertebrae followed by bracing for 16 weeks gave good results. Long term follow up has shown persistent stability and alignment. Both Taylor braces without groin straps, and Plastazote-lined polythene braces were used depending on the level of the fracture and both proved satisfactory.

\section{Conclusion}

A non-operative regimen of treatment of fractured thoracic and lumbar vertebrae as described is an alternative to prolonged immobilisation or early operative fusion.

\section{References}

BEDBROOK GM 1975 Treatment of thoracolumbar dislocation and fractures with paraplegia. Clinical Orthopaedics and Related Research 112:27-49

BEDBROOK GM 1985 A balanced viewpoint in the early management of patients with spinal injuries who have neurological damage. Paraplegia 23:8-15

FRANKEL HL et al. 1969 The value of postural reduction in the initial management of closed injuries of the spine with paraplegia and tetraplegia. Paraplegia Part 17:179-192

Garzione JE, Clifton SJ 1976 Orthosis for geriatric patients with vertebral fracture. Physical Therapy 56:1034-1035

Guttman L 1976 Spinal Cord Injuries: Comprehensive Management and Research. 2nd Ed., Blackwell Scientific Publications, 6, Oxford

HÄRKÖNEN M et al. 1969 Fractures of the thoracic spine. Clinical and radiological results in ninety eight patients. Archives of Orthopaedic and Traumatic Surgery 94:179-84

NAGEL DA et al. 1981 Stability of the upper lumbar spine following progressive disruptions and the application of individual internal and external fixation devices. Fournal of Bone and foint Surgery 63-A:62-70

RYAN MD, TAYLOR TKF 1982 The early management of thoraco-lumbar fractures by an open reduction and internal fixation. Australian and New Zealand fournal of Surgery 52:236

STAUFFER ES 1974 Orthotics for spinal injuries. Clinical Orthopaedics and Related Research 102:92-99 